\title{
Overexpression of PD-L2 is associated with shorter relapse-free survival in patients with malignant salivary gland tumors
}

\author{
Hyeyoon Chang' \\ Jung Sun Kim² \\ Yoon Ji Choi ${ }^{3}$ \\ Jae-Gu Cho ${ }^{4}$ \\ Jeong-Soo $\mathrm{Woo}^{4}$ \\ Aeree Kim' \\ Jun Suk Kim ${ }^{5}$ \\ Eun Joo Kang ${ }^{5}$
}

'Department of Pathology, Korea University Guro Hospital, Korea University College of Medicine, Seoul, Korea; ${ }^{2}$ Division of Oncology, Department of Internal Medicine, Korea University Ansan Hospital, Korea University College of Medicine, Seoul, Korea; ${ }^{3}$ Division of Oncology, Department of Internal Medicine, Korea University Anam Hospital, Korea University College of Medicine, Seoul, Korea; ${ }^{4}$ Department of Otorhinolaryngology-Head and Neck Surgery, Korea University Guro Hospital, Korea University College of Medicine, Seoul, Korea; ${ }^{5}$ Division of Oncology, Department of Internal Medicine, Korea University Guro Hospital, Korea University College of Medicine, Seoul, Korea
Correspondence: Eun Joo Kang Division of Oncology, Department of Internal Medicine, Korea University Guro Hospital, Korea University College of Medicine, 148 Gurodong-ro, Guro-gu, Seoul, 08308, Korea

Tel +82 22626306 I

Email kkangjul I@naver.com

\author{
This article was published in the following Dove Press journal: \\ OncoTargets and Therapy \\ 13 June 2017 \\ Number of times this article has been viewed
}

\begin{abstract}
Objectives: PD-1/PD-L1 and CTLA-4 have been investigated and are thought to play an important role in tumor evasion. This study aimed to investigate expression patterns of immunerelated molecules, and their clinical impacts in malignant salivary gland tumors.
\end{abstract}

Patients and methods: We performed immunohistochemical staining for PD-L1, PD-L2, CTLA-4, PD-1, and CD8 ${ }^{+}$tumor-infiltrating lymphocytes in 70 malignant salivary gland tumors. Protein expression was assessed by H-score by multiplying the staining intensity by the percentage of cells with positive staining.

Results: The tumors comprised mucoepidermoid carcinomas (38.6\%), adenoid cystic carcinomas (21.4\%), salivary duct carcinomas (15.7\%), and others. In malignant salivary gland tumors, PD-L2 expression was high, while expression of PD-L1 was relatively low in terms of the percentage of positively stained cells and the staining intensity. In univariate analysis, PD-L2 expression ( $\mathrm{H}$-score $<1$ vs $\geq 1$ ), PD-1 (H-score $<1$ vs $\geq 1$ ), and CD $8^{+}$tumor-infiltrating lymphocytes ( $\mathrm{H}$-score $<1$ vs $\geq 1$ ) were significant prognostic factors. In multivariate analysis, low PD-L2 expression ( $\mathrm{H}$-score $<1$ ) was independently associated with shorter relapse-free survival (hazard ratio $=6.514 ; 95 \%$ confidence interval, $1.2-36.2 ; P=0.032$ ).

Conclusion: In summary, PD-L2 is potentially an important biomarker in malignant salivary gland tumors, especially in regard to relapse.

Keywords: malignant salivary gland tumors, PD-L1, PD-L2, CTLA-4, tumor-infiltrating lymphocytes, relapse-free survival

\section{Introduction}

Salivary gland tumors are a complex disease entity, encompassing eleven benign and 20 malignant tumors according to the 2017 World Health Organization classification. ${ }^{1}$ Malignant salivary gland tumors occur rarely, with an overall global prevalence of $1.2 / 100,000 .^{2}$ Owing partly to the low prevalence (less than $1 \%$ of all cancers) of these tumors and the diverse pathological subtypes, currently available information regarding predictive and prognostic clinicopathological factors for survival is insufficient. Complete surgical excision is the primary treatment strategy for patients with salivary gland tumors, followed by radiotherapy or chemoradiotherapy in patients with malignant histology such as adenoid cystic carcinoma or salivary duct carcinoma, especially with features associated with high risk of recurrence. In advanced cases, chemotherapy may be used for systemic control, and radiotherapy, particle therapy including proton and carbon ion therapy, or concurrent chemoradiotherapy may facilitate palliation or local symptomatic control. ${ }^{3}$ 
At present, the immune checkpoints, which involve the PD-1 receptor and its ligand, PD-L1, have become a global focus in oncology. Recent studies have reported great advances in the treatment of various cancers with immunotherapeutic agents such as immune checkpoint inhibitors, and these agents are being used in the clinical setting in the treatment of malignant melanoma, non-small cell lung cancer (NSCLC), and other malignancies. ${ }^{4,5}$

PD-1, also known as CD279, is an inhibitory receptor expressed by activated $\mathrm{T}$ cells which inhibits T-cell receptormediated lymphocyte proliferation, resulting in immune evasion by human cancer cells. ${ }^{6}$ PD-L1 (also known as B7-H1 or CD274) is the most well-known PD-1 ligand, and has been extensively studied. ${ }^{7}$ PD-1/PD-L1 expression has been found to show an association with poor prognosis and poor prognostic clinicopathological features in several human solid cancers, including renal cell carcinoma, ${ }^{8} \mathrm{NSCLC},{ }^{9}$ and breast, ${ }^{10}$ gastric, ${ }^{11}$ hepatocellular, ${ }^{12}$ and ovarian carcinomas. ${ }^{13}$ PD-L2 (also known as CD273 or B7-DC) is another PD-1 ligand, and also plays a role in regulating tumor immunity. Although several studies have been performed to investigate this role, the available information is insufficient to allow any conclusions to be drawn. ${ }^{14,15}$ The CD28/CTLA-4 pathway is another well-established pathway involved in tumor immunity. CTLA-4 first competes with $\mathrm{CD} 28$, which is expressed on $\mathrm{CD} 4^{+}$and $\mathrm{CD} 8^{+}$ $\mathrm{T}$ cells, then binds with two main ligands, CD80 and CD86, which are present on antigen presenting cells. The interaction of CTLA-4 with CD80/86 generates intrinsic inhibitory signals and prevents $\mathrm{T}$ cell activation, thereby causing activation of tumor cells. ${ }^{16}$ Anti-CTLA-4 antibody has also shown clinical activity and is used for treating malignant melanoma. ${ }^{5}$

Although extensive studies have been conducted in various cancers, information regarding these immune-related markers in salivary gland tumors is still scarce. Therefore, we performed immunohistochemical (IHC) analysis of PD-1, PD-L1, PD-L2, and CTLA-4 in malignant salivary gland tumors to evaluate their expression patterns and clinical impact. Moreover, infiltration of cells such as CD $8^{+}$, PD-1 tumor-infiltrating lymphocytes (TILs) into the tumor microenvironment is known to affect tumor immunity and the response to therapeutic interventions. ${ }^{17}$ Therefore, we also evaluated the expression of $\mathrm{CD} 8^{+}$and PD- $1^{+}$TILs in these tumors by IHC, and evaluated the correlations between clinicopathological factors and prognosis.

\section{Patients and methods}

\section{Patients}

Samples of malignant salivary gland tumors from patients diagnosed between 2001 and 2015 at the Korea University
Guro Hospital were included in this study. All patients whose tissues were used in this research had provided written informed consent for their use in future research. All patients had undergone an excision and/or neck lymph node dissection as an initial treatment. Clinical data including age, sex, recurrence, metastasis, and treatment were obtained on reviewing medical records and tumor registry information. Pathological and histological reports were assessed by an experienced pathologist, Chang HY, who was blinded to the clinical information, to confirm the diagnosis and evaluate the pathological factors including diagnosis, TNM (tumor, lymph node, metastases) stage, histological grade, and lymphovascular and perineural invasion. Tumors were staged in accordance with the criteria outlined in the 8th edition of the American Joint Committee on Cancer TNM staging manual. ${ }^{18}$ Tumor histology was based on the World Health Organization classification system. ${ }^{1}$ The study protocol was approved by the Institutional Review Board of Korea University Guro Hospital (KUGH15053-001).

\section{Construction of the tissue microarray (TMA) blocks}

All hematoxylin and eosin ( $\mathrm{H}$ and $\mathrm{E})$-stained tissue slides were assessed by an experienced pathologist, Chang HY. The most representative tumor section was identified via microscopic examination and $2 \mathrm{~mm}$ plugs were obtained from formalin-fixed, paraffin-embedded tumor tissue blocks. The plugs were then embedded in a recipient block to construct the TMA. For cases with sufficient tumor area, two cores were sampled: one from the center and one from the periphery of the tumor. Two cores could be sampled in half of all cases. In another half of cases, one core was achieved from the tumor center. Normal tissue, distant from the tumor, was also sampled as a negative control. $\mathrm{H}$ and E-stained sections of the TMA were reviewed via microscopic examination to confirm the suitability of the sampled areas.

\section{IHC analysis}

IHC staining for PD-L1, PD-L2, CTLA-4, PD-1, and CD8 ${ }^{+}$ TILs in the TMA sections was performed using the standard streptavidin-biotin complex method. We used the automated BOND-MAX staining system (Leica Microsystems, Wetzlar, Germany). After IHC staining, the TMA slides were scanned using a slide scanner (ScanScope ${ }^{\circledR}$ CS; Aperio, Vista, CA, USA). PD-1, PD-L1, and PD-L2 IHC showed a membranous pattern and CD8 showed a cytoplasmic and membranous pattern. CTLA-4 IHC showed a cytoplasmic pattern. Using the Aperio ScanScope ${ }^{\circledR}$ digital image analysis program (Aperio ScanScope ${ }^{\circledR}$; Aperio Technologies, CA, USA), digital image 
analysis was performed. We used optimal protocols for the evaluation of nuclear, cytoplasmatic, and membranous tumor biomarkers. The percentage of immunoreactive cells, cell numbers, and intensity scores were generated automatically for each core sample. The staining intensity was graded from 0 to 3 ( 0 , negative; 1 , weak; 2 , moderate; 3 , strong) and the percentage of positively stained cells $(0 \%-100 \%)$ was calculated. Then, H-score was calculated to evaluate the tumor expression of proteins by multiplying staining intensity with the percentage of positively stained cells. ${ }^{19}$ Primary antibodies and dilutions were as follows: antiPD-1 antibody (1:100, clone NAT105, mouse monoclonal; Abcam, Cambridge, MA, USA), anti-PD-L1 antibody (1:100, rabbit polyclonal; Abcam), anti-PD-L2 antibody (1:200, clone 176611, mouse monoclonal; R\&D Systems, Inc., Minneapolis, MN, USA), anti-CTLA-4 antibody (1:50, clone F-8, mouse monoclonal; Santa Cruz Biotechnology Inc., Dallas, TX, USA), anti-CD8 antibody (1:200, clone 4B11, mouse monoclonal; Leica Microsystems).

\section{Statistical analysis}

To analyze the association between the level of immunerelated protein expressions and clinicopathological variables, chi-square test was used. Pearson's correlation test was performed to analyze the association between immune-related protein expressions. Relapse-free survival (RFS) was defined as the time from the initiation of treatment, such as surgery, to the first locoregional or distant recurrence. Overall survival (OS) was defined as the time from the initiation of treatment to death due to any cause. RFS and OS were evaluated using the Kaplan-Meier method. The differences between the curves were analyzed using the log-rank test. After univariate analyses using the Kaplan-Meier method, variables which were significantly associated with poor survival time (variables with $P<0.05$ ) were selected and a Cox proportional hazards regression was conducted for multivariate analyses by the "enter" method, to identify variables which could predict RFS or OS. The Wilcoxon signed-rank test was used to compare variables between primary and recurrent tumor tissue. All statistical analyses were performed using IBM SPSS Statistics for Windows, version 21.0 (IBM Corporation, Armonk, NY, USA) with $P<0.05$ as the threshold of statistical significance.

\section{Results}

\section{Patient characteristics}

IHC data were available for 71 of the 89 tumor samples. Clinicopathological information was available for 70 cases. The median estimated follow-up duration was 37.6 months (range, 10.4-181.8 months). The median age of all patients was 54 years (range, 11-84 years). Forty patients $(57.1 \%)$ were women and 30 patients $(42.9 \%)$ were men. Mucoepidermoid carcinoma was the most common subtype (27 patients, $38.6 \%$ ), followed by adenoid cystic carcinoma (15 patients, $21.4 \%$ ), salivary duct carcinoma (eleven patients, 15.7\%), and acinic cell carcinoma (seven patients, $10.0 \%$ ). Squamous cell carcinoma (three patients, $4.3 \%$ ), adenocarcinoma (two patients, $2.9 \%$ ), carcinoma ex-pleomorphic adenoma (two patients, 2.9\%), epithelialmyoepithelial carcinoma (two patients, 2.9\%), and undifferentiated carcinoma (one patient, 1.4\%) were also included among the tumor types in the study. The parotid gland was the most common primary tumor site $(52.9 \%)$, followed by the submandibular gland $(12.9 \%)$. For the initial treatment, surgery was performed in $64(91.4 \%)$ patients. Among the 70 patients, recurrence was confirmed in 17 patients $(24.3 \%)$. Patient characteristics are shown in Table 1.

\section{Expression patterns of markers and clinicopathological characteristics according to histology}

Representative microscopic images of IHC staining are shown in Figure 1. PD-L1 was not well expressed in any salivary gland tumor samples. The median $\mathrm{H}$-score for PD-L1 was 0 (range, 0-272.6). For PD-L2 and CTLA-4, relatively strong and diffuse uptake was observed. The median H-score for PD-L2 was 52.6 (range, 0-265.8), and the median H-score for CTLA-4 was 33.7 (range, 0-265.8).

Table 2 shows the expression rate of the molecular markers in three tumor subtypes: adenoid cystic carcinomas $(\mathrm{N}=15)$, salivary duct carcinomas $(\mathrm{N}=11)$, and mucoepidermoid carcinomas ( $\mathrm{N}=27)$. PD-L1 was not strongly expressed in any of these three tumor subtypes. PD-L1 negativity was observed in $73.3 \%$ of adenoid cystic carcinomas, $54.5 \%$ of salivary duct carcinomas, and $74.1 \%$ of mucoepidermoid carcinomas. However, PD-L2 and CTLA-4 were expressed in all three tumor types. PD-L2 positivity was observed in $86.7 \%, 63.6 \%$, and $63.0 \%$ of cases of adenoid cystic carcinoma, salivary duct carcinoma, and mucoepidermoid carcinoma, respectively. Focusing only on staining intensity, PD-L2 expression was high, particularly in adenoid cystic carcinoma, with $46.7 \%$ of adenoid cystic carcinoma cases demonstrating a PD-L2 intensity score of $3+$. In terms of CTLA-4, $46.7 \%$ of cases of adenoid cystic carcinoma, and $81.8 \%$ of cases of salivary duct carcinoma displayed positive CTLA- 4 expression scores $>2+$. Figure 2 shows the proportion of staining intensity for PD-L1, PD-L2, and CTLA-4 in adenoid cystic carcinoma and salivary duct carcinoma. 
Table I Baseline characteristics for all patients with salivary gland tumors

\begin{tabular}{|c|c|c|}
\hline Parameters & $\mathbf{N}$ & $\%$ \\
\hline \multicolumn{3}{|l|}{ Sex } \\
\hline Male & 30 & 42.9 \\
\hline Female & 40 & 57.1 \\
\hline Age, median (range) & $54(\mathrm{II}-84)$ & \\
\hline \multicolumn{3}{|l|}{ Primary site } \\
\hline Parotid gland & 37 & 52.9 \\
\hline Submandibular gland & 9 & 12.9 \\
\hline Sublingual gland & I & 1.4 \\
\hline Hard palate & 5 & 7.1 \\
\hline Soft palate & 1 & 1.4 \\
\hline Nasal cavity & 5 & 7.1 \\
\hline Maxilla & 4 & 5.7 \\
\hline Oral cavity & 8 & 11.4 \\
\hline \multicolumn{3}{|l|}{ Histology } \\
\hline Adenoid cystic carcinoma & 15 & 21.4 \\
\hline Salivary duct carcinoma & 11 & 15.7 \\
\hline Mucoepidermoid carcinoma & 27 & 38.6 \\
\hline Acinic cell carcinoma & 7 & 10 \\
\hline Adenocarcinoma & 2 & 2.9 \\
\hline Carcinoma ex-pleomorphic adenoma & 2 & 2.9 \\
\hline Squamous cell carcinoma & 3 & 4.3 \\
\hline Epithelial-myoepithelial carcinoma & 2 & 2.9 \\
\hline Undifferentiated carcinoma & 1 & 1.4 \\
\hline \multicolumn{3}{|l|}{ T stage } \\
\hline 1 & 28 & 40 \\
\hline 2 & 18 & 25.7 \\
\hline 3 & 14 & 20 \\
\hline 4 & 10 & 14.3 \\
\hline \multicolumn{3}{|l|}{$\mathrm{N}$ stage } \\
\hline 0 & 61 & 87.1 \\
\hline I & 6 & 8.6 \\
\hline 2 & 3 & 4.3 \\
\hline \multicolumn{3}{|l|}{ M stage } \\
\hline 0 & 68 & 97.1 \\
\hline I & 4 & 5.7 \\
\hline \multicolumn{3}{|l|}{ Initial stage } \\
\hline 1 & 28 & 40 \\
\hline 2 & 19 & 27.1 \\
\hline 3 & 12 & 17.1 \\
\hline 4 & 13 & 18.6 \\
\hline \multicolumn{3}{|l|}{ Treatment } \\
\hline Surgery & 45 & 64.3 \\
\hline Surgery + adjuvant RT & 18 & 25.7 \\
\hline Surgery + adjuvant CCRT & 2 & 2.9 \\
\hline CCRT & 1 & 1.4 \\
\hline RT alone & 3 & 4.3 \\
\hline No treatment & 1 & 1.4 \\
\hline \multicolumn{3}{|l|}{ Recurrence } \\
\hline No & 53 & 75.7 \\
\hline Yes & 17 & 24.3 \\
\hline
\end{tabular}

Abbreviations: CCRT, concurrent chemoradiotherapy; $\mathrm{T}$, tumor; $\mathrm{N}$, lymph nodes; $M$, metastases; $R T$, radiotherapy.

The proportion of PD-1 expression was low and the intensity was relatively low. The median H-score for PD-1 expression was 1.2 (range, 0-21.7). The median $\mathrm{H}$-score for $\mathrm{CD}^{+} \mathrm{TIL}$ expression was 8.41 (0-98.4). For PD-1, 80\% of patients with adenoid cystic carcinoma demonstrated low expression, with
H-score $<1$. In salivary duct carcinoma, $27.3 \%$ of patients had $\mathrm{H}$-score $<1$, while $18.5 \%$ of patients with mucoepidermoid carcinoma had H-score $<1$. For $\mathrm{CD} 8^{+} \mathrm{TILs}$, an H-score $\geq 1$ was observed in $81.8 \%$ of cases of salivary duct carcinoma, and $92.6 \%$ of cases of mucoepidermoid carcinoma.

\section{Association between clinicopathological variables and immune-related proteins}

PD-L1 and CD8 expressions were strongly associated with tumor size. They showed inverse results. Low expression of PD-L1 was associated with smaller tumor size $(<4 \mathrm{~cm})$, while high expression of CD8 expression showed an association with smaller tumor size. CTLA-4 showed an association with the presence of vascular emboli. Statistically significant associations were not observed for PD-L2 and PD1 expressions with clinicopathological variables (Table 3).

\section{Survival and recurrence}

For all patients, the median OS was 75.9 months (95\% confidence interval [CI], 59.0-92.7). The median OS was 73.1 months (95\% CI, 54.0-92.2) for patients with adenoid cystic carcinoma and 75.9 months (95\% CI, 51.0-100.7) for patients with mucoepidermoid carcinoma. The median OS for patients with salivary duct carcinoma was 37.5 months (95\% CI, 5.1-70.0). In univariate analysis with the clinicopathological variables, only initial M stage status (M0 vs M1) was significant $(P=0.008)$. The median RFS of all patients was 22.4 months (95\% CI, 15.6-29.1). To investigate prognostic factors, we classified protein expression status by cut-off point of H-score; $<1$ vs $\geq 1$. We applied the same cut-off point (H-score $<1$ vs $\geq 1$ ) to all markers for reliable and reproducible results, and further application to other cancers. In univariate analysis, PD-L2 expression (H-score $<1$ vs $\geq 1$ ), PD-1 (H-score $<1$ vs $\geq 1$ ), and CD $8^{+}$TILs (H-score $<1$ vs $\geq 1$ ) were significant prognostic factors (Table 4). Multivariate analysis using a Cox proportional hazards regression model, revealed that PD-L2 expression with an $\mathrm{H}$-score $<1$ was independently associated with shorter RFS (hazard ratio $=6.514 ; 95 \% \mathrm{CI}$ 1.2-36.2; $P=0.032$ ) (Table 5). The RFS curve according to the PD-L2 expression is shown in Figure 3.

\section{Protein expression in primary and recurrent tumors}

Among the 17 patients who experienced recurrence, tumor tissue from both the primary site and the site of recurrence was obtained for seven patients. Comparison of protein expression between the primary tumor and the recurrent tumor was performed using the Wilcoxon signed-rank test. 

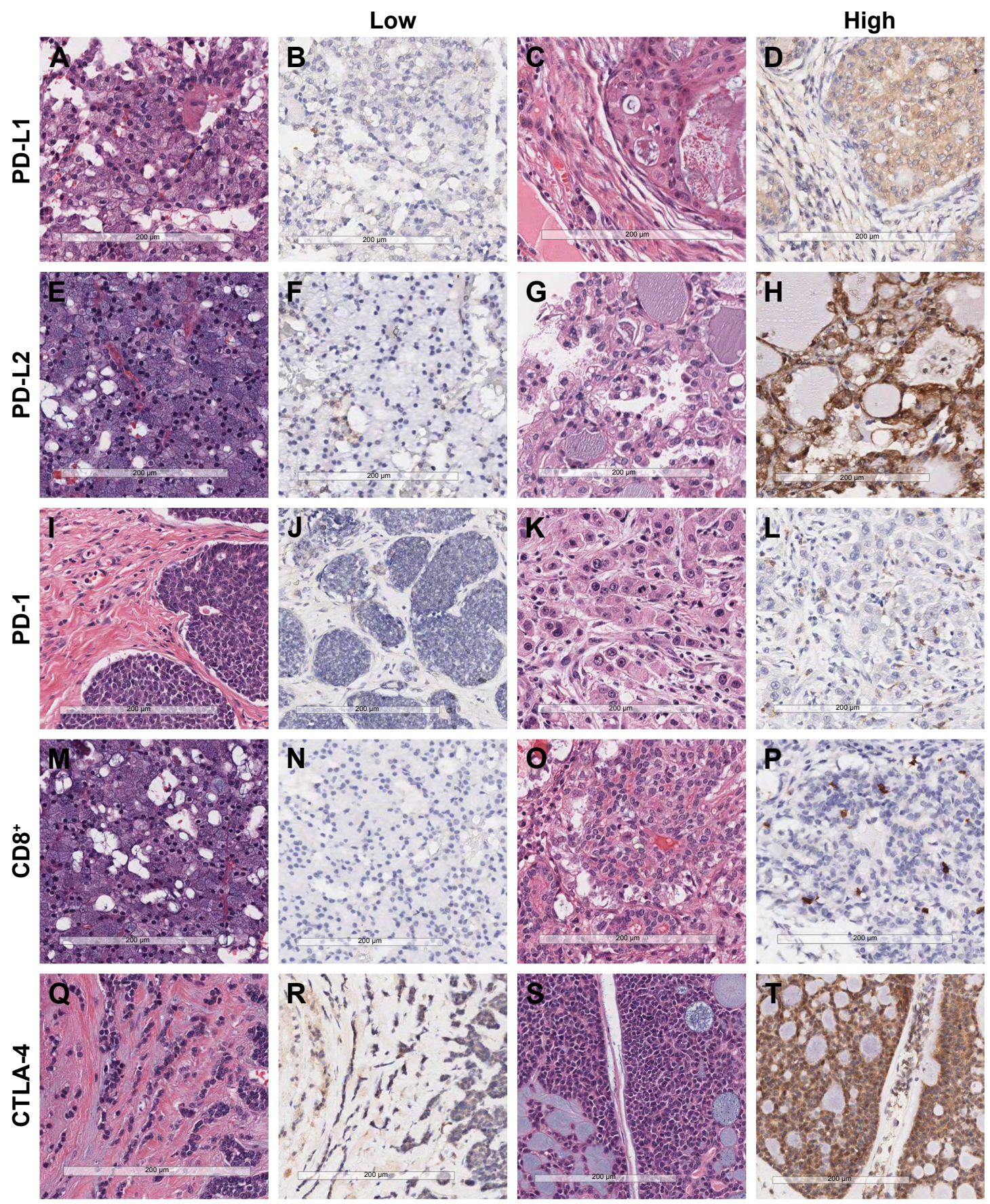

Figure I Representative examples of hematoxylin and eosin staining and immunohistochemical staining for PD-LI, PD-L2, PD-I, CD8 ${ }^{+}$tumor-infiltrating lymphocytes, and CTLA-4 in malignant salivary gland tumors $(\times 200)$.

Notes: Low PD-LI (A, B, acinic cell carcinoma), high PD-LI (C, D, mucoepidermoid carcinoma), low PD-L2 (E, F, acinic cell carcinoma), high PD-L2 (G, H, acinic cell carcinoma), low PD-I (I, J, adenoid cystic carcinoma), high PD-I (K, L, salivary duct carcinoma), low CD8 $8^{+}$tumor-infiltrating lymphocytes (M, N, acinic cell carcinoma), high CD8 ${ }^{+}$tumor-infiltrating lymphocytes (O, P, epithelial-myoepithelial carcinoma), low CTLA-4 (Q, R, adenoid cystic carcinoma), and high CTLA-4 expression (S, T, adenoid cystic carcinoma). Scale bars at the bottom of the figure indicate $200 \mu \mathrm{m}$.

No difference in protein expression was observed between the two sites for any of the variables (Table 6).

\section{Discussion}

Among the salivary gland tumors, malignant salivary gland tumors including adenoid cystic carcinoma and salivary duct carcinoma have high recurrence rates and the ability to metastasize to other organs. However, there are only a few chemotherapeutic agents that have shown effects for systemic control. ${ }^{20,21}$

In the present study, we assessed the expression patterns of the immune-related markers in malignant salivary gland tumors. Although numerous studies have been performed 
Table 2 Molecular marker expression in adenoid cystic carcinoma, salivary duct carcinoma, and mucoepidermoid carcinoma

\begin{tabular}{|c|c|c|c|c|c|c|}
\hline \multirow{2}{*}{$\begin{array}{l}\text { H-score } \\
\text { PD-LI }\end{array}$} & \multicolumn{2}{|c|}{$\begin{array}{l}\text { Adenoid cystic } \\
\text { carcinoma } \\
(\mathrm{N}=15)\end{array}$} & \multicolumn{2}{|c|}{$\begin{array}{l}\text { Salivary duct } \\
\text { carcinoma } \\
(\mathbf{N}=\text { II) }\end{array}$} & \multicolumn{2}{|c|}{$\begin{array}{l}\text { Mucoepidermoid } \\
\text { carcinoma } \\
(\mathbf{N}=\mathbf{2 7})\end{array}$} \\
\hline & & & & & & \\
\hline$<1$ & 11 & 73.3 & 6 & 54.5 & 20 & 74.1 \\
\hline$\geq 1$ & 4 & 26.7 & 5 & 45.5 & 7 & 25.9 \\
\hline \multicolumn{7}{|l|}{ PD-L2 } \\
\hline$<1$ & 2 & 13.3 & 4 & 36.4 & 10 & 37 \\
\hline$\geq 1$ & 13 & 86.7 & 7 & 63.6 & 17 & 63 \\
\hline \multicolumn{7}{|l|}{ CTLA-4 } \\
\hline$<1$ & 4 & 26.7 & 2 & 18.2 & 7 & 25.9 \\
\hline$\geq 1$ & 11 & 73.3 & 9 & 81.8 & 20 & 74.1 \\
\hline \multicolumn{7}{|l|}{ PD-I } \\
\hline$<1$ & 12 & 80 & 3 & 27.3 & 5 & 18.5 \\
\hline$\geq 1$ & 3 & 20 & 8 & 72.7 & 22 & 81.5 \\
\hline \multicolumn{7}{|l|}{ CD8+ TILs } \\
\hline$<1$ & 9 & 60 & 2 & 18.2 & 2 & 7.4 \\
\hline$\geq 1$ & 6 & 40 & 9 & 81.8 & 25 & 92.6 \\
\hline
\end{tabular}

Note: $\mathrm{H}$-score, percentage of positive cells multiplied by intensity. Abbreviations: H-score, Histoscore; TILs, tumor-infiltrating lymphocytes. to investigate immune-related biomarker expression in various tumors, there is no standardized evaluation method or antibody for PD-L1, PD-L2, or CTLA-4 expression. In previous studies investigating the efficacy of anti-PD-1 inhibitor in NSCLC, PD-L1 positivity was selected as a predictive marker and defined in accordance with the percentage of all tumor cells with positive expressions, regardless of staining intensity. ${ }^{22}$ In the study of atezolizumab (Tecentriq $^{\circledR}$, Hoffman-La Roche, Basel, Switzerland), an anti-PD-L1 antibody, IHC staining was assessed in tumor cells and tumor-infiltrating immune cells. ${ }^{23}$ However, many other studies have investigated staining intensity, incorporating the proportion of cells with positive expression for evaluation of PD-L1 expression. ${ }^{24,25}$ In addition, defining positivity, staining intensity, or cut-off values between low and high expression have differed among the previous studies. In the present study, we used a digital image analyzer for assessment of staining intensity and calculated the
A
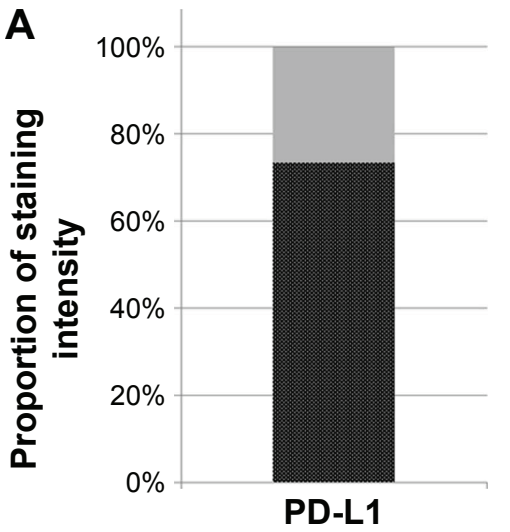

PD-L2 3+ $\square$ PD-L1 2+

PD-L1 1+ PD-L1 0

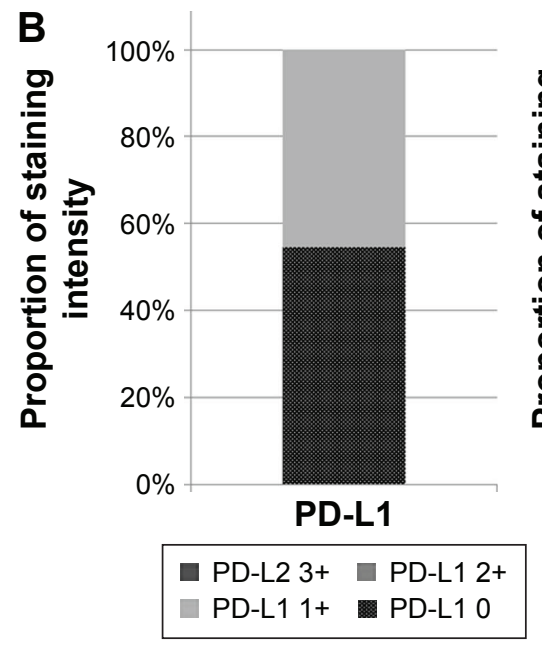

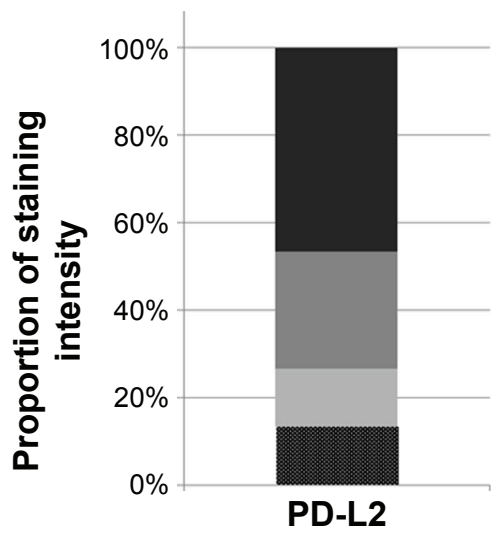

PD-L2 3+ $\quad$ PD-L2 2+ PD-L2 1+ PD-L2 0

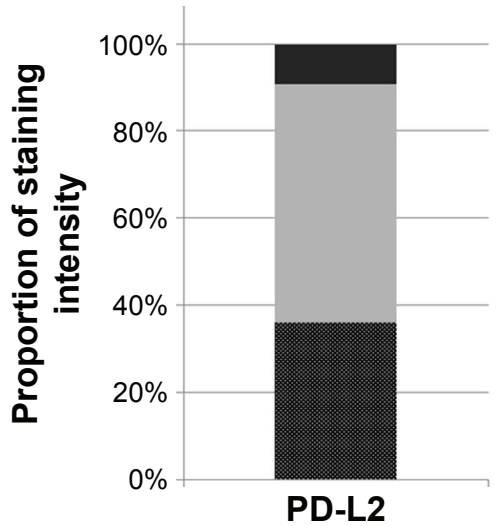

PD-L2 3+ PD-L2 2+ PD-L2 1+ PD-L2 0

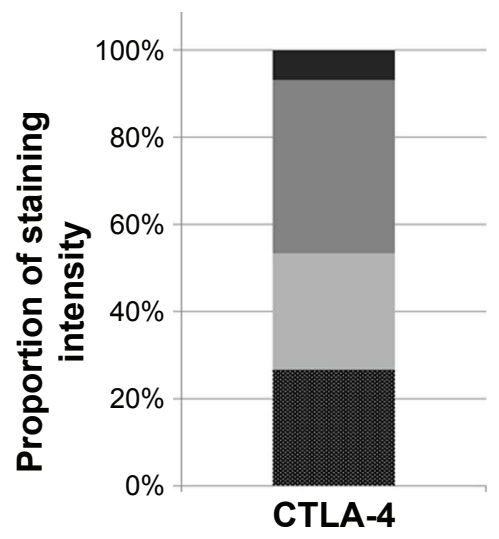

CTLA-4 3+ CTLA-4 2+

CTLA-4 1+ CTLA-4 0

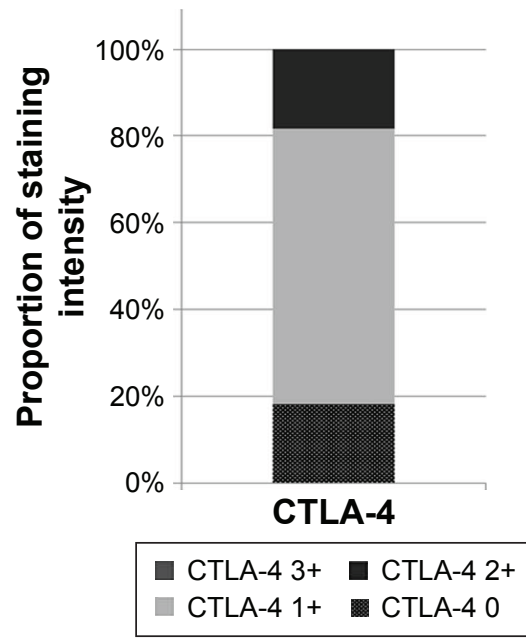

Figure 2 The proportion of staining intensity of PD-LI, PD-L2, and CTLA-4 expression in adenoid cystic carcinoma and salivary duct carcinoma. Notes: (A) Adenoid cystic carcinoma; (B) salivary duct carcinoma. 


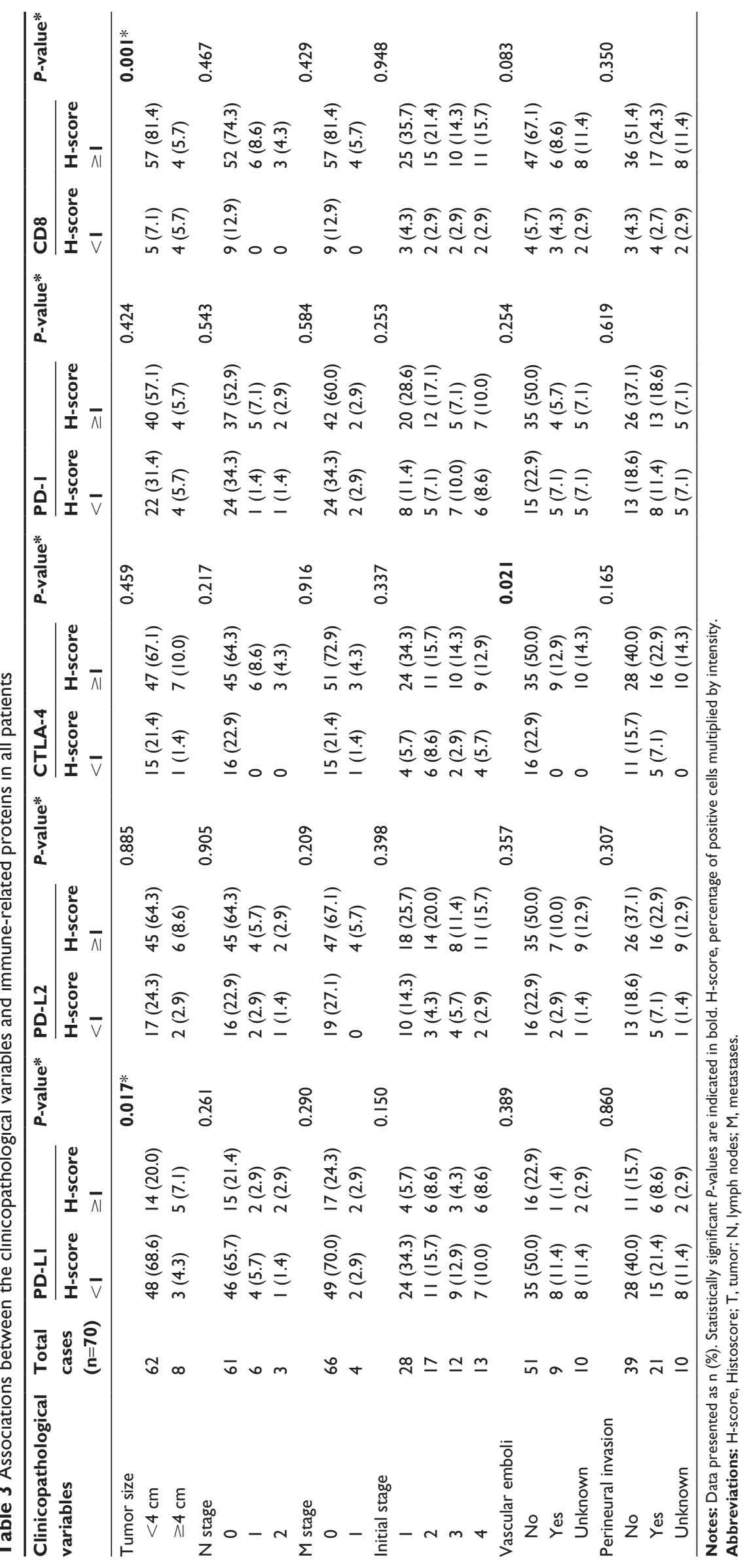


Table 4 Univariate analysis for relapse-free survival and overall survival in all patients

\begin{tabular}{|c|c|c|c|c|}
\hline & \multicolumn{2}{|l|}{ RFS } & \multicolumn{2}{|l|}{ os } \\
\hline & $95 \% \mathrm{Cl}$ & $P$-value & $95 \% \mathrm{Cl}$ & $P$-value \\
\hline PD-LI & 25.9 vs 20.9 & 0.644 & 75.9 vs 73.1 & 0.853 \\
\hline $\begin{array}{l}(\mathrm{H} \text {-score }<\mathrm{I} \text { vs } \geq \mathrm{I}) \\
\text { PD-L2 }\end{array}$ & 10.0 vs 26.0 & 0.004 & 63.5 vs 77.3 & 0.782 \\
\hline$(\mathrm{H}$-score $<\mathrm{I}$ vs $\geq \mathrm{I})$ & & & & \\
\hline $\begin{array}{l}\text { CTLA-4 } \\
(\mathrm{H} \text {-score }<\mathrm{I} \text { vs } \geq \mathrm{I})\end{array}$ & I5.I vs 22.4 & 0.26 & - vs 75.9 & 0.782 \\
\hline PD-I & 26.4 vs 21.3 & 0.044 & 73.1 vs 75.9 & 0.772 \\
\hline $\begin{array}{l}(\mathrm{H} \text {-score }<\mathrm{I} \text { vs } \geq \mathrm{I}) \\
\mathrm{CD} 8^{+}\end{array}$ & 29.2 vs 21.3 & 0.037 & 75.9 vs 73.1 & 0.731 \\
\hline$(\mathrm{H}$-score $<\mathrm{I}$ vs $\geq \mathrm{I})$ & & & & \\
\hline
\end{tabular}

Note: $\mathrm{H}$-score, percentage of positive cells multiplied by intensity.

Abbreviations: $\mathrm{Cl}$, confidence interval; $\mathrm{H}$-score, Histoscore; $\mathrm{RFS}$, relapse-free survival; OS, overall survival.

percentage of all cells with positive expression. Therefore, the proportion of cells with positive expression in this study was calculated among all tumor cells and tumor-infiltrating immune cells in TMA slides. In addition, IHC scoring of tumor cells and immune cells by eye, by a pathologist, is time consuming and bears a risk of lack of reproducibility among pathologists. Therefore, we performed our analysis using a digital image analyzer for both convenience and reproducibility.

The overall expression of PD-L1 was low in all salivary gland tumors in this study. However, the overall expression of PD-L2 showed a higher intensity and proportion than PD-L1 in all histological subtypes. Moreover, low expression of PD-L2 was associated with significantly shorter RFS in all salivary gland tumors. Nevertheless, the clinical significance of PD-L2 expression in cancer has been less explored compared with PD-L1 expression. PD-L2 expression has been reported in various cell types including tumor cells, dendritic cells, and subsets of B and T cells. ${ }^{15}$ PD-L2 plays an important role in immunity, such as the modulation of T cell responses via binding to PD-1, similar to PD-L1. However, the molecular mechanisms of interaction between PD-1 and PD-L2 are known to be distinct from those of PD-1 and PD-L1. Although there have been extensive studies and therapeutics developed against PD-1/PD-L1, PD-L2 has not

Table 5 Multivariate analysis for relapse-free survival in all patients

\begin{tabular}{llll}
\hline & \multicolumn{2}{l}{ RFS } & \\
\cline { 2 - 4 } & HR & $\mathbf{9 5 \%} \mathbf{~ C l}$ & P-value \\
\hline PD-L2 $($ H-score $<$ I vs $\geq I)$ & $6.5 I 4$ & $1.173-36.186$ & $\mathbf{0 . 0 3 2}$ \\
PD-I $($ H-score $<$ I vs $\geq I)$ & 0.706 & $0.184-2.716$ & 0.613 \\
CD8 $($ H-score vs $\geq I)$ & 0.242 & $0.046-I .263$ & 0.092
\end{tabular}

Notes: Statistically significant $P$-values are indicated in bold. $\mathrm{H}$-score, percentage of positive cells multiplied by intensity.

Abbreviations: HR, hazard ratio; H-score, Histoscore; RFS, relapse-free survival; $\mathrm{Cl}$, confidence interval.



Figure 3 Relapse-free survival curve for all salivary gland tumors according to PD-L2 expression.

Abbreviation: $\mathrm{H}$-score, Histoscore.

been an object of focus as a therapeutic target in cancer, in comparison to PD-L1.

The majority of studies have reported a correlation between survival and PD-L1 expression, but much less so for PD-L2. In addition, prognostic correlations with PD-L2 expression have been inconsistent according to the tumor histology. Ohigashi et al reported worse survival in PD-L2-negative cases in patients with esophageal cancer. ${ }^{26}$ However, positive PD-L2 expression was significantly associated with shorter progression-free survival in renal cell carcinoma. ${ }^{27}$ In the present study, low PD-L2 expression with an $\mathrm{H}$-score $<1$ was significantly associated with shorter RFS in all salivary gland tumors. In adenoid cystic carcinoma, poorer RFS was shown in cases of patients with $\mathrm{H}$-score $<1$. In salivary duct carcinoma, which also has highly malignant characteristics, a similar trend was observed for RFS, although there was no statistical significance. Since the number of cases of each histological type was not sufficient in this study, proving statistical significance for each type of tumor histology was difficult. Therefore, more extensive studies with larger numbers of cases regarding PD-L2 are required to define its role in each histological type of salivary gland tumor.

Among the diverse histology of salivary gland tumors, adenoid cystic carcinoma and salivary duct carcinoma are important because of their malignant clinical characteristics. Adenoid cystic carcinoma has a clinical course that may range from very indolent to extensive multiple organ metastases. Since adenoid cystic carcinoma does not respond well to cytotoxic chemotherapy, a new effective therapeutic agent 
Table 6 Expression of PD-LI, PD-L2, CTLA-4, PD-I, and CD8 $8^{+}$tumor-infiltrating lymphocytes in primary and recurrent tumors

\begin{tabular}{|c|c|c|c|c|c|c|c|c|c|c|}
\hline \multirow[t]{2}{*}{ Patient } & \multicolumn{2}{|c|}{ PD-LI (H-score) } & \multicolumn{2}{|c|}{ PD-L2 (H-score) } & \multicolumn{2}{|c|}{ CTLA-4 (H-score) } & \multicolumn{2}{|c|}{ PD-I (H-score) } & \multicolumn{2}{|c|}{ CD8 (H-score) } \\
\hline & $\begin{array}{l}\text { Primary } \\
\text { site }\end{array}$ & $\begin{array}{l}\text { Site of } \\
\text { recurrence }\end{array}$ & $\begin{array}{l}\text { Primary } \\
\text { site }\end{array}$ & $\begin{array}{l}\text { Site of } \\
\text { recurrence }\end{array}$ & $\begin{array}{l}\text { Primary } \\
\text { site }\end{array}$ & $\begin{array}{l}\text { Site of } \\
\text { recurrence }\end{array}$ & $\begin{array}{l}\text { Primary } \\
\text { site }\end{array}$ & $\begin{array}{l}\text { Site of } \\
\text { recurrence }\end{array}$ & $\begin{array}{l}\text { Primary } \\
\text { site }\end{array}$ & $\begin{array}{l}\text { Site of } \\
\text { recurrence }\end{array}$ \\
\hline A & 6.6 & 0 & 100.6 & 58.6 & 1.1 & 0 & 0.5 & 0 & 0.9 & 0 \\
\hline B & 0 & 1.7 & 37.7 & 63.9 & 30.6 & 5.2 & 0.8 & 0.8 & 3 & 5.2 \\
\hline C & 0 & 7.1 & 48.7 & 37.7 & 41.5 & 3 & 2.2 & 5.2 & 22.5 & 3 \\
\hline$D$ & 0 & 0 & 131.4 & 89.5 & 0 & 3 & 0 & 0 & 1 & 3 \\
\hline$E$ & 15.3 & 0 & 230.2 & 60.3 & 0 & 5.4 & 0.5 & 5 & 12.3 & 5.4 \\
\hline $\mathrm{F}$ & 3 & 0 & 44.3 & 41.2 & 81.5 & 76.5 & 3.8 & 0.9 & 27.3 & 76.5 \\
\hline G & 0 & 3.5 & 78.5 & 68.5 & 48.5 & 9.3 & 3.4 & 1.5 & 5.6 & 9.3 \\
\hline$P$-value & 0.753 & & 0.091 & & 0.5 & & 0.735 & & 0.735 & \\
\hline
\end{tabular}

Note: $\mathrm{H}$-score, percentage of positive cells multiplied by intensity.

Abbreviation: $\mathrm{H}$-score, Histoscore.

is necessary for patients with multiple organ metastases. As salivary duct carcinoma also has a very poor prognosis, and there is a lack of effective therapeutic agents, the investigation of new agents is urgently required. Therefore, in the present study, we particularly focused on adenoid cystic carcinoma and salivary duct carcinoma to determine whether the application of immune checkpoint inhibitors such as PD-L1, PD-1, or CTLA-4 inhibitors, which have shown great success recently in the treatment of many other cancers, would be possible in these tumor types. According to the results of this study, it is suggested that PD-L2 is an important investigational and therapeutic target candidate. Future studies focusing on PD-L2 expression and target agents should be promising.

Sometimes, it is not possible in clinical practice to retrieve tumor specimens from primary sites or sites of recurrence. Since the expression of immune-related markers is considered to be influenced by various conditions of the tumor microenvironment, differences in expression of these markers, according to the tumor site or the time at which the tumor specimen was retrieved, have been explored. In a previous study of PD-L1 in primary tumors and recurrent or metastatic tumors, concordance was observed between the primary and metastatic lesions. ${ }^{28}$ In the analysis of the expression rate of the molecular markers in primary and recurrent tumors from seven patients in this study, no statistically significant differences were identified. Nonetheless, there is a lack of information regarding discrepancies between molecular markers in primary and metastatic or recurrent salivary gland tumors, and studies with larger numbers of cases will provide more validated results.

This study has the following limitations. First, the use of a TMA to analyze protein expression could cause a sampling error because TMA represents only a small portion of whole tumor tissue. To overcome this limitation, we tried to achieve multiple cores from tumor center and invasive front.
However, we achieved a single core in each sample in half of all cases, and we obtained two cores in the remaining half. Therefore, caution is needed not to over-interpret results of this study. Second, the histological subtypes are diverse, and we were able to include only a small sample size for each histological type; therefore, the tumor samples cannot be characterized as simply salivary gland tumors. However, this is the first study investigating expression patterns of immunerelated markers and their prognostic value in salivary gland tumors. Since the number of patients with salivary gland tumors is low, clinical studies are not focused on these tumors. However, considering the subset of patients who have a poor prognosis, investigation of potential biomarkers is important. In addition, the application of anti-PD-1 treatment, or future treatment focusing on anti-PD-L2 agents, must be evaluated for patients with salivary gland tumors.

\section{Conclusion}

In malignant salivary gland tumors, PD-L2 expression was high, and low expression of PD-L2 showed a negative prognostic impact in terms of RFS. Further studies focusing on PD-L2 are important in malignant salivary tumors.

\section{Acknowledgments}

The biospecimens for this study were provided by the Korea University Guro Hospital of National Biobank, a member of the National Biobank of Korea.

The study was supported by a grant received by DrEun Joo Kang from Korea University, Seoul, Korea (K1422231).

\section{Disclosure}

The authors report no conflicts of interest in this work.

\section{References}

1. El-Naggar AK, Chan JK, Grandis JR, Takata T, Slootweg PJ, editors. WHO Classification of Head and Neck Tumours. 4th ed. IARC: Lyon; 2017. 
2. Surveillance, Epidemiology, and End Results (SEER) Program. SEER*Stat Database: Incidence - SEER 9. Available from: https:// seer.cancer.gov/. Accessed April 23, 2017.

3. Pfister DG, Spencer S, Brizel DM, et al. Head and neck cancers, version 1 2015. J Natl Compr Canc Netw. 2015;13(7):847-855.

4. Swaika A, Hammond WA, Joseph RW. Current state of anti-PD-L1 and anti-PD-1 agents in cancer therapy. Mol Immunol. 2015; 67(2 Pt A):4-17.

5. Hodi FS, O'Day SJ, McDermott DF, et al. Improved survival with ipilimumab in patients with metastatic melanoma. $N$ Engl $J$ Med. 2010;363(8):711-723.

6. Pardoll DM. Spinning molecular immunology into successful immunotherapy. Nat Rev Immunol. 2002;2(4):227-238.

7. Freeman GJ, Long AJ, Iwai Y, et al. Engagement of the PD-1 immunoinhibitory receptor by a novel $\mathrm{B} 7$ family member leads to negative regulation of lymphocyte activation. $J$ Exp Med. 2000;192(7):1027-1034.

8. Thompson RH, Dong H, Lohse CM, et al. PD-1 is expressed by tumorinfiltrating immune cells and is associated with poor outcome for patients with renal cell carcinoma. Clin Cancer Res. 2007;13(6):1757-1761.

9. Zhang Y, Huang S, Gong D, Qin Y, Shen Q. Programmed death-1 upregulation is correlated with dysfunction of tumor-infiltrating CD8+ T lymphocytes in human non-small cell lung cancer. Cell Mol Immunol. 2010;7(5):389-395.

10. Muenst S, Soysal SD, Gao F, Obermann EC, Oertli D, Gillanders WE. The presence of programmed death 1 (PD-1)-positive tumor-infiltrating lymphocytes is associated with poor prognosis in human breast cancer. Breast Cancer Res Treat. 2013;139(3):667-676.

11. Wu C, Zhu Y, Jiang J, Zhao J, Zhang XG, Xu N. Immunohistochemical localization of programmed death-1 ligand-1 (PD-L1) in gastric carcinoma and its clinical significance. Acta Histochem. 2006; 108(1):19-24.

12. Shi F, Shi M, Zeng Z, et al. PD-1 and PD-L1 upregulation promotes $\mathrm{CD} 8(+) \mathrm{T}$-cell apoptosis and postoperative recurrence in hepatocellular carcinoma patients. Int J Cancer. 2011;128(4):887-896.

13. Hamanishi J, Mandai M, Iwasaki M, et al. Programmed cell death 1 ligand 1 and tumor-infiltrating CD8+ T lymphocytes are prognostic factors of human ovarian cancer. Proc Natl Acad Sci U SA. 2007;104(9): 3360-3365.

14. Baptista MZ, Sarian LO, Derchain SF, Pinto GA, Vassallo J. Prognostic significance of PD-L1 and PD-L2 in breast cancer. Hum Pathol. 2016; 47(1):78-84.

15. Rozali EN, Hato SV, Robinson BW, Lake RA, Lesterhuis WJ. Programmed death ligand 2 in cancer-induced immune suppression. Clin Dev Immunol. 2012;2012:656340.
16. Gardner D, Jeffery LE, Sansom DM. Understanding the CD28/CTLA-4 (CD152) pathway and its implications for costimulatory blockade. Am J Transplant. 2014;14(9):1985-1991.

17. Ji RR, Chasalow SD, Wang L, et al. An immune-active tumor microenvironment favors clinical response to ipilimumab. Cancer Immunol Immunother. 2012;61(7):1019-1031.

18. Amin MB, Edge S, Greene F, et al, editors. AJCC Cancer Staging Manual. 8th ed. New York: Springer; 2017.

19. McCarty KS Jr, Miller LS, Cox EB, Konrath J, McCarty KS Sr. Estrogen receptor analyses. Correlation of biochemical and immunohistochemical methods using monoclonal antireceptor antibodies. Arch Pathol Lab Med. 1985;109(8):716-721.

20. Guzzo M, Di Palma S, Grandi C, Molinari R. Salivary duct carcinoma: clinical characteristics and treatment strategies. Head Neck. 1997;19(2): 126-133.

21. Dillon PM, Chakraborty S, Moskaluk CA, Joshi PJ, Thomas CY. Adenoid cystic carcinoma: a review of recent advances, molecular targets, and clinical trials. Head Neck. 2016;38(4):620-627.

22. Herbst RS, Baas P, Kim DW, et al. Pembrolizumab versus docetaxel for previously treated, PD-L1-positive, advanced non-small-cell lung cancer (KEYNOTE-010): a randomised controlled trial. Lancet. 2016;387(10027):1540-1550.

23. Fehrenbacher L, Spira A, Ballinger M, et al. Atezolizumab versus docetaxel for patients with previously treated non-small-cell lung cancer (POPLAR): a multicentre, open-label, phase 2 randomised controlled trial. Lancet. 2016;387(10030):1837-1846.

24. Igarashi T, Teramoto K, Ishida M, Hanaoka J, Daigo Y. Scoring of PD-L1 expression intensity on pulmonary adenocarcinomas and the correlations with clinicopathological factors. ESMO Open. 2016;1(4): e000083.

25. Katsuya Y, Horinouchi H, Asao T, et al. Expression of programmed death 1 (PD-1) and its ligand (PD-L1) in thymic epithelial tumors: Impact on treatment efficacy and alteration in expression after chemotherapy. Lung Cancer. 2016;99:4-10.

26. Ohigashi Y, Sho M, Yamada Y, et al. Clinical significance of programmed death-1 ligand-1 and programmed death-1 ligand-2 expression in human esophageal cancer. Clin Cancer Res. 2005;11(8):2947-2953.

27. Shin SJ, Jeon YK, Kim PJ, et al. Clinicopathologic analysis of PD-L1 and PD-L2 expression in renal cell carcinoma: association with oncogenic proteins status. Ann Surg Oncol. 2016;23(2):694-702.

28. Patel SP, Kurzrock R. PD-L1 expression as a predictive biomarker in cancer immunotherapy. Mol Cancer Ther. 2015;14(4):847-856.
OncoTargets and Therapy

\section{Publish your work in this journal}

OncoTargets and Therapy is an international, peer-reviewed, open access journal focusing on the pathological basis of all cancers, potential targets for therapy and treatment protocols employed to improve the management of cancer patients. The journal also focuses on the impact of management programs and new therapeutic agents and protocols on
Dovepress

patient perspectives such as quality of life, adherence and satisfaction. The manuscript management system is completely online and includes a very quick and fair peer-review system, which is all easy to use. Visit http://www.dovepress.com/testimonials.php to read real quotes from published authors. 